\title{
GROWTH OF THE EIGENSOLUTIONS OF LAPLACIANS ON RIEMANNIAN MANIFOLDS II: POSITIVITY OF THE INITIAL ENERGY
}

\author{
WENCAI LIU
}

\begin{abstract}
In this paper, energy function is used to investigate the eigen-solutions of $-\Delta u+V u=\lambda u$ on the Riemannian manifolds. We give a new way to prove the positivity of the initial energy of energy function, which leads to a simple way to obtain the growth of eigen-solutions.
\end{abstract}

\section{INTRODUCTION AND MAIN RESULTS}

The present paper is the second in a series 23] that discusses the growth of eigen-solutions of Laplacian on connected non-compact complete Riemannian manifolds.

Let $(M, g)$ be a connected $n$-dimensional noncompact complete Riemannian manifold $(n \geq$ 2 ). The Laplace-Beltrami operator on $(M, g)$ is essentially self-adjoint on $C_{0}^{\infty}(M)$. We denote the self-adjoint extension by $\Delta$ (Laplacian). Assume there exists $U \subset M$ such that $M \backslash U$ is connected and the induced outward normal exponential map $\exp _{\partial U}^{\perp}: N^{+}(\partial U) \rightarrow M-\bar{U}$ is diffeomorphism, where $N^{+}(\partial U)=\{v \in T(\partial U) \mid v$ is outward normal to $\partial U\}$. Let $r$ be the distance function from $\partial U$ defined on the end $M-\bar{U}$. This is the same setting as in [18, 20, 23].

An interesting problem is to investigate if the eigenvalues can be embedded into the essential spectrum of Laplacian on Riemannian manifolds. We focus on two popular manifoldsasymptotically flat case and asymptotically hyperbolic case, which are distinguished by the radial curvature $K_{\text {rad }}(r)$. Radial curvature is a very important quantity in spectral geometry [8]. Roughly speaking, asymptotically flat manifold is characterized by the radial curvature $K_{\text {rad }}(r)=O\left(r^{-\alpha}\right)$ with some $\alpha>0$, and respectively asymptotically hyperbolic manifold is characterized by the radial curvature $K_{\mathrm{rad}}(r)=-1+O\left(r^{-\alpha}\right)$. This topic was heavily studied by various authors in geometry and analysis $2,3,5-7,10-13,17,19-22,24]$.

For the asymptotically hyperbolic case, the sharp spectral transition is given by Kumura [18]. He excludes eigenvalues greater than $\frac{(n-1)^{2}}{4}$ under the assumption that $K_{\text {rad }}(r)=-1+$

$o\left(r^{-1}\right)$, and also constructs a manifold for which exact an eigenvalue $\frac{(n-1)^{2}}{4}+1$ is embedded into its essential spectrum $\left[\frac{(n-1)^{2}}{4}, \infty\right)$ with the radial curvature $K_{\mathrm{rad}}(r)=-1+O\left(r^{-1}\right)$. Jitomirskaya and Liu constructed examples which show that dense eigenvalues and singular continuous spectrum can be embedded into the essential spectrum [14, 15].

For the asymptotically flat case, the authors in [3 -5, 7] showed the absence of positive eigenvalues of Laplacian on complete Riemannian manifold with a pole, under the assumptions more or less like $\left|K_{\text {rad }}(r)\right| \leq \frac{\delta}{1+r^{2}}$ for small $\delta$. Kumura obtained a similar result (see Remark 1.6) which works for more general manifolds [20].

We should mention that the spectral theory of free Laplacian on asymptotically hyperbolic and flat manifolds is more or less parallel to that of Schrödinger operators with decaying potentials [1]. We refer the readers to our previous paper [23].

Kumura [18, 20] achieved his goals by the careful study of energy function of eigen-solutions directly. Our inspiration comes from Kato [16]. His basic idea is (four steps): construct energy 
function for eigen-equation; set up the positivity of initial energy; prove the monotonicity of energy function with respect to $r$; obtain the growth of eigen-solution. In our previous paper 23], we have already used such scheme to set up the growth of eigen-solutions by a universal construction of energy functions. As an application, we showed the absence of eigenvalues in certain circumstances, which improved Kumura's sharp results [18] mentioned before. However, we rely on strong convexity of Hessian of distance function $r$ to set up the positivity (or non-negativity) of the initial energy. Such strong convexity is a big obstacle for the asymptotically flat manifolds, which prevents us from getting sharp bounds. Since the energy function on the manifold (even on the Euclidean space in higher dimensions) is not the simple sum of potential energy and kinetic energy any more, the non-negativity of $-\Delta$ can not lead to the positivity (or non-negativity) of initial energy. Notice that the positivity of initial energy for simply connected manifolds is trivial because the energy function we constructed is zero at the initial point $r=0$ automatically. This explains why it is easier to study the spectral theory of Laplacian on simply connected manifolds in some sense.

The novelty of this paper is to give an effective and new way to verify the positivity (or nonnegativity) of the initial energy for non-simply connected manifolds. The idea is to increase the potential energy via modifying the potential without breaking the scheme (four steps) in [16, 23]. We show that if the positivity of the initial energy fails, by solving a ODE problem, the weakly exponential decay $\left(e^{-c \sqrt{r}}\right.$ with some $\left.c>0\right)$ of the eigen-solution can be established. By using a class of test functions introduced in 20] and developing the methods of [20], we prove that $u$ is exponentially decaying $\left(e^{-c r}\right.$ with some $\left.c>0\right)$ and then is a trivial solution by additional arguments. As one of the applications, the results in [20] are recovered without the extra assumptions on the Ricci curvature. To our best knowledge, this is a new way to prove the positivity of initial energy. We only give one kind of such theorems here. The gradient version and mixed version can also be established by combining with the arguments in [23]. Moreover, we believe our method has a wider applicability.

Another approach to investigate the absence of embedded eigenvalues is based on Mourre type commutator estimates and super-exponential decay estimates of a priori eigenfunction. See [10] and references therein. Ito and Skibsted obtained some criteria for the absence of embedded eigenvalues for both asymptotically flat and hyperbolic Riemannian Laplacians [10], which are similar to the results in the present paper and our previous paper [23. We will say more later. Ito and Skibsted also set up the scattering theory for Riemannian Laplacians in their series of papers $[\underline{9}, 11-13]$.

Let $g$ be the metric and $\nabla$ be the covariant derivative. Denote Hessian of $r$ by $\nabla d r$. Let

$$
\begin{aligned}
& M(r)=M(r ; u)=\left(\int_{|r(x)|=r}|u(x)|^{2} d x\right)^{\frac{1}{2}}, \\
& N(r)=N(r ; u)=\left(\int_{|r(x)|=r}\left|\frac{\partial u}{\partial r}\right|^{2} d x\right)^{\frac{1}{2}} .
\end{aligned}
$$

Our main result in the present paper is

Theorem 1.1. Let the potential $V(r)=V_{1}(r)+V_{2}(r)$. Suppose

$$
\left|V_{1}(r)\right|=\frac{o(1)}{r},\left|V_{2}(r)\right|=o(1),\left|\frac{\partial V_{2}}{\partial r}\right|=\frac{o(1)}{r},
$$

as $r$ goes to infinity. Suppose

$$
\liminf _{r \rightarrow \infty}[r \nabla d r-a \hat{g}] \geq 0,
$$


for some $a>0$, where $\hat{g}=g-d r \otimes d r$, and

$$
\limsup _{r \rightarrow \infty} r\left|\Delta r-b-\frac{c}{r}\right| \leq \delta,
$$

for some constants $b, c, \delta$. Suppose

$$
\mu>\delta, 2 a>\mu+\delta
$$

and

$$
\lambda>\frac{b^{2}}{4}+\frac{\delta^{2} b^{2}}{\mu^{2}-\delta^{2}} .
$$

Then we have

$$
\liminf _{r \rightarrow \infty} r^{\mu}\left[M(r)^{2}+N(r)^{2}\right]=\infty .
$$

Based on the universal Theorem 1.1, we have several interesting corollaries. Letting $b=0$ in Theorem 1.1], we have

Corollary 1.2. Let the potential $V(r)=V_{1}(r)+V_{2}(r)$. Suppose

$$
\left|V_{1}(r)\right|=\frac{o(1)}{r},\left|V_{2}(r)\right|=o(1),\left|\frac{\partial V_{2}}{\partial r}\right|=\frac{o(1)}{r}
$$

as $r$ goes to infinity. Suppose

$$
\liminf _{r \rightarrow \infty}[r \nabla d r-a \hat{g}] \geq 0,
$$

for some $a>0$, where $\hat{g}=g-d r \otimes d r$, and

$$
\limsup _{r \rightarrow \infty} r\left|\Delta r-\frac{c}{r}\right| \leq \delta
$$

for some constants $c, \delta$. Suppose

$$
\delta<\min \{a, 1\} .
$$

Then $-\Delta+V$ admits no positive eigenvalue.

Corollary 1.3. Suppose

$$
\frac{a}{r} \hat{g} \leq \nabla d r \leq \frac{b}{r} \hat{g}
$$

for large $r$, where $a>0$ and $b>0$ are constants satisfying

$$
a \leq b \quad \text { and } \quad \frac{n+1}{n-1} a>b .
$$

Let $\lambda>0$ and $u$ be a nontrivial solution to the eigen-equation

$$
\Delta u+\lambda u=0
$$

Then for any

$$
\mu>\frac{n-1}{2}(b-a)
$$

we have

$$
\liminf _{r \rightarrow \infty} r^{\mu}\left[M(r)^{2}+N(r)^{2}\right]=\infty
$$

\footnotetext{
${ }^{1}$ By the fact that $\Delta+V$ is essentially selfadjoint, we have $\nabla u \in L^{2}(M)$ if the eigensolution $u \in L^{2}(M)$.
} 
Proof. By the fact that $\Delta r$ is the trace of $\nabla d r$, one has

$$
\frac{(n-1) a}{r} \leq \Delta r \leq \frac{(n-1) b}{r} .
$$

So

$$
\left|\Delta r-\frac{(b-a)(n-1)}{2 r}\right| \leq \frac{\delta}{r}
$$

where $\delta=\frac{(b-a)(n-1)}{2}$. Let $s$ satisfy $\mu>s>\delta$ and be close to $\delta$ so that

$$
2 a>\delta+s
$$

by (41). By Theorem 1.1, one has

$$
\liminf _{r \rightarrow \infty} r^{s}\left[M(r)^{2}+N(r)^{2}\right]=\infty
$$

which implies

$$
\liminf _{r \rightarrow \infty} r^{\mu}\left[M(r)^{2}+N(r)^{2}\right]=\infty
$$

Letting $\mu=1$ in Corollary 1.3 , we can get the following result.

Corollary 1.4. Suppose

$$
\frac{a}{r} \hat{g} \leq \nabla d r \leq \frac{b}{r} \hat{g}
$$

for large $r$, where $a>0$ and $b>0$ are constants satisfying

$$
a \leq b \text { and } \frac{n+1}{n-1} a>b .
$$

Suppose

$$
\frac{n-1}{2}(b-a)<1 .
$$

Then $\sigma(-\Delta)=\sigma_{\mathrm{ess}}(-\Delta)=[0, \infty)$ and $\sigma_{\mathrm{p}}(-\Delta)=\emptyset$.

Similarly, we have

Corollary 1.5. Let $\kappa>0$. Suppose

$$
\left(\kappa+\frac{a}{r}\right) \hat{g} \leq \nabla d r \leq\left(\kappa+\frac{b}{r}\right) \hat{g}
$$

for large $r$, where $a>0$ and $b>0$ are constants satisfying

$$
a \leq b \text { and }(n-1)(b-a)<2 .
$$

Let

$$
E_{0}=\frac{\kappa^{2}(n-1)^{2}}{4}+\frac{(n-1)^{4} \kappa^{2}(b-a)^{2}}{4-(n-1)^{2}(b-a)^{2}} .
$$

Then $-\Delta$ does not have eigenvalue larger than $E_{0}$.

Remark 1.6. - We used the fact that $\sigma_{\mathrm{ess}}(-\Delta)=\left[\frac{c^{2}}{4}, \infty\right)$ if $\lim \Delta r=c$ [6, 17] and $-\Delta$ is a non-negative operator.

- Corollaries 1.3 and 1.4 were obtained by Kumura [20] with some extra assumptions on Ricci curvature. 
A result similar to Theorem 1.1] has been already obtained in [10, 23] and similar Corollaries 1.3 and 1.4 have also been already obtained. Here, we want to say more about the theorems in [10, 23] and the present paper. We will use Corollaries 1.4 and 1.5] as an example first. Under the same assumptions of Corollary 1.5, it was shown $-\Delta$ does not have eigenvalue larger than $E_{2}([10$, Corollary 2.4]), where

$$
E_{2}=\frac{\kappa^{2}(n-1)^{2}}{2(2-(n-1)(b-a))} .
$$

Under the same assumptions of Corollary 1.5 it was shown $-\Delta$ does not have eigenvalue larger than $E_{1}$ (see Corollary 1.3 and Remark 1.4 in $[23]$ ), where

$$
E_{1}=\frac{\kappa^{2}(n-1)^{2}}{4}+\frac{(n-1)^{4} \kappa^{2}(b-a)^{2}}{4\left(4-(n-1)^{2}(b-a)^{2}\right)} .
$$

Thus we have

Corollary 1.4 : For $\kappa=0$, the assumptions in the present paper are stronger than those in [10]. Thus the result in [10] is best.

Corollary 1.5 : For $\kappa>0$, all the assumptions in [10, Theorem 1.5], 23, Theorem 1.1] and present paper are exactly the same. The bound $E_{1}$ in [23] is the best, that is $E_{1} \leq E_{0}$ and $E_{1} \leq E_{2}$. If $b-a$ is small (large), the bound $E_{0}$ in the present paper is better (worse) than that in [10].

It is not just the corollaries in the three papers are comparable, the main theorems- 10 , Theorem 1.5], 23, Theorem 1.1] and Theorem [1.1 are also comparable. Theorem 1.5 in 10] was formulated in a different way, which is involved into the derivatives of $\Delta r$ and $r(x)$ is not necessary to be the distance function. By direct modifications of our arguments, a similarly generalized version may also be obtained.

Suppose $b>0$. Theorem 1.1 implies (letting $\mu=1$ ), if $\delta<1,-\Delta$ does not have eigenvalue larger than $E_{0}$, where

$$
E_{0}=\frac{b^{2}}{4}+\frac{\delta^{2} b^{2}}{1-\delta^{2}}
$$

Under the assumptions of Theorem 1.1. Ito and Skibsted obtained that 2 if $\delta<1,-\Delta$ does not have eigenvalue larger than $E_{2}$

$$
E_{2}=\frac{b^{2}}{4(1-\delta)} .
$$

Under the assumptions of Theorem 1.1 with $a \geq 2$, the bound obtained in [23] is best among the three papers. More precisely, $-\Delta$ does not have eigenvalue larger than $E_{1}$

$$
E_{1}=\frac{b^{2}}{4}+\frac{\delta^{2} b^{2}}{4\left(1-\delta^{2}\right)}
$$

for $\delta<1$.

Without the extra assumption $a \geq 2$, whether the bound in [10] or in the present paper is better depends on the size of the perturbation $\delta$ ( $\frac{1}{3}$ is the threshold).

Letting $b=0$ in Theorem 1.1, we have

Corollary 1.7. Suppose

$$
\nabla d r=\left(\frac{a}{r}+\frac{o(1)}{r}\right) \hat{g}
$$

\footnotetext{
${ }^{2}$ Let $c_{1}=2-2 \delta, \rho_{1}=2\left(\delta+r\left(\Delta r-b-\frac{c}{r}\right)\right), \rho_{2}=0, \rho_{3}=2+2 r b+2 c-2 \delta$ and $c_{2}>0$ be arbitrarily small in Theorem 1.5 in [10]. Such setting is optimal under the assumptions of Theorem 1.1
} 
for some $a>0$. Let the potential $V(r)=V_{1}(r)+V_{2}(r)$. Suppose

$$
\left|V_{1}(r)\right|=\frac{o(1)}{r},\left|V_{2}(r)\right|=o(1),\left|\frac{\partial V_{2}}{\partial r}\right|=\frac{o(1)}{r} .
$$

Then operator $-\Delta+V$ does not have positive eigenvalue.

Remark 1.8. Corollary 1.7 works for in particular Euclidean space $\left(\nabla d r=\frac{1}{r} \hat{g}\right)$, covering a well known result.

Corollary 1.9. Let $M^{n}$ be a complete Riemannian manifold with a pole. Suppose the radial curvature satisfies $K_{\mathrm{rad}}(r) \leq \frac{\delta_{n}}{1+r^{2}}$, for sufficiently small $\delta_{n}$. Then $M$ admits no eigenvalue.

Remark 1.10. - Corollary 1.9 was proved by Donnelly and Garofalo [5]. A weaker version (curvature condition $0 \leq K_{\mathrm{rad}}(r) \leq \frac{\delta_{n}}{1+r^{2}}$ ) was proved by Escobar-Freire [7]. Corollary 1.9 follows from Theorem 1.1 and comparison theorem. We gave up the details here. See Lemma 1.2 [7], and Propositions 2.1, 2.2 in [20] for comparison theorem.

- By checking our proof, Corollary 1.9 also holds if we replace the radial curvature with Ricci curvature of the direction $\nabla r$.

Now the only task is to prove Theorem 1.1. The rest of the paper is organized as follows: In $\S 2$, we present some basic knowledge. In $\S 3$, we will give the construction of energy functions and derive their derivatives. In $\S 4$, we prove the exponential decay of the eigensolution. In $\S 5$, we establish the positivity of the initial energy corresponding to the energy function and give the proof of Theorem 1.1 .

\section{Preliminaries AND Derivative lemma}

We recall some notations in 23] first. Let $S_{t}=\{x \in M: r(x)=t\}, \omega \in S_{r}$ and $x \in M$. Thus $(r, \omega)$ is a local coordinate system for $M$ 3. Let $\langle\cdot, \cdot\rangle$ be the metric on Riemannian manifold.

Let $\hat{L}=e^{\rho} L e^{-\rho}$, where $L=-\Delta+V$. We draw the graph to describe the relation.

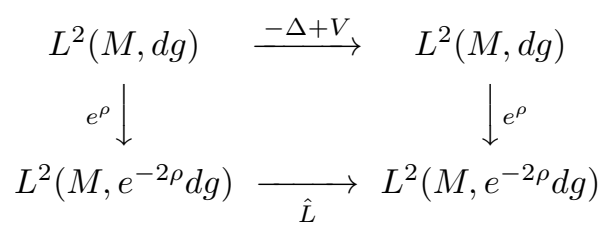

Let

Then, one has

$$
v=e^{\rho} u .
$$

and

$$
\nabla u=-\rho^{\prime} e^{-\rho} v \nabla r+e^{-\rho} \nabla v
$$

$$
\Delta u=\operatorname{div} \nabla u=e^{-\rho} \Delta v-2 \rho^{\prime} e^{-\rho} \frac{\partial v}{\partial r}+\left(\rho^{2}-\rho^{\prime \prime}-\rho^{\prime} \Delta r\right) e^{-\rho} v .
$$

So the eigen-equation becomes

$$
-\Delta v+2 \rho^{\prime} \frac{\partial v}{\partial r}+\left(V_{1}+V_{2}+V_{0}\right) v=\lambda v
$$

where

$$
V_{0}=\rho^{\prime} \Delta r+\rho^{\prime \prime}-\rho^{2}
$$

\footnotetext{
${ }^{3} \omega$ depends on $r$. We ignore the dependence for simplicity.
} 
Let $2 \rho^{\prime}=b+\frac{c}{r}$ and $r\left(\Delta r-b-\frac{c}{r}\right)=\bar{\delta}(r)$. Then

$$
V_{0}=\frac{b^{2}}{4}+\frac{b c}{2 r}+\frac{b \bar{\delta}}{2 r}+\frac{O(1)}{r^{2}} .
$$

We should mention that $O(1)$ and $o(1)$ in the proof only depend on constants in the assumptions of Theorem 1.1 .

Now we always assume $u$ is a nontrivial solution of $-\Delta u+V u=\lambda u$, where $V=V_{1}+V_{2}$. Let

$$
v=e^{\rho} u,
$$

and

$$
v_{m}=r^{m} v
$$

with $m \geq 0$. By (5), we get the equation of $v_{m}$,

(6) $\Delta v_{m}-\left(\frac{2 m}{r}+2 \rho^{\prime}\right) \frac{\partial v_{m}}{\partial r}+\left(\frac{m(m+1)}{r^{2}}+\frac{m}{r}\left(2 \rho^{\prime}-\Delta r\right)-V_{0}-V_{1}-V_{2}+\lambda\right) v_{m}=0$.

Lemma 2.1. [18, 23] Let $X$ be a vector field. Then

$$
\frac{\partial}{\partial r} \int_{S_{r}}\langle X, \nabla r\rangle e^{-2 \rho} d x=\int_{S_{r}}\left(\operatorname{div} X-2 \rho^{\prime}\langle X, \nabla r\rangle\right) e^{-2 \rho} d x
$$

Lemma 2.2. [18, 23]

$$
\frac{\partial}{\partial r} \int_{S_{r}} f e^{-2 \rho} d x=\int_{S_{r}}\left[\frac{\partial f}{\partial r}+f\left(\Delta r-2 \rho^{\prime}\right)\right] e^{-2 \rho} d x .
$$

Lemma 2.3. Let $q_{0}=\lambda-V_{0}-V_{1}-V_{2}+\frac{m(m+1)}{r^{2}}+\frac{m}{r}\left(2 \rho^{\prime}-\Delta r\right)$. Suppose

$$
\liminf _{r \rightarrow \infty} \int_{S_{r}} r^{1-2 m}\left|\frac{\partial v_{m}}{\partial r} v_{m}\right| e^{-2 \rho} d x=0 .
$$

Then the following holds

$$
\begin{aligned}
\int_{r>t} r^{1-2 m}\left[\left|\nabla v_{m}\right|^{2}-q_{0} v_{m}^{2}\right] e^{-2 \rho} d x= & -\frac{1}{2} \frac{d}{d t}\left(t^{1-2 m} \int_{S_{t}} v_{m}^{2} e^{-2 \rho} d x\right)-\frac{1}{2} \int_{S_{t}} r^{-2 m}(2 m-1) v_{m}^{2} e^{-2 \rho} d x \\
& +\frac{1}{2} \int_{S_{t}} t^{1-2 m}\left(\Delta r-2 \rho^{\prime}\right) v_{m}^{2} e^{-2 \rho} d x-\int_{r>t} r^{-2 m} \frac{\partial v_{m}}{\partial r} v_{m} e^{-2 \rho} d x .
\end{aligned}
$$

Proof. By (8), there exists a sequence $r_{j}$ going to infinity such that

$$
\int_{S_{r_{j}}} r^{1-2 m}\left|\frac{\partial v_{m}}{\partial r} v_{m}\right| e^{-2 \rho} d x=o(1) .
$$

By Lemma 2.2. one has

$$
\begin{aligned}
-\frac{1}{2} \frac{d}{d t}\left(t^{1-2 m} \int_{S_{t}} v_{m}^{2} e^{-2 \rho} d x\right)= & -\frac{1}{2}(1-2 m) t^{-2 m} \int_{S_{t}} v_{m}^{2} e^{-2 \rho} d x-\frac{1}{2} t^{1-2 m} \int_{S_{t}}\left(\Delta r-2 \rho^{\prime}\right) v_{m}^{2} e^{-2 \rho} d x \\
& -t^{1-2 m} \int_{S_{t}} \frac{\partial v_{m}}{\partial r} v_{m} e^{-2 \rho} d x .
\end{aligned}
$$


WENCAI LIU

Multiplying $r^{1-2 m} v_{m} e^{-2 \rho}$ on both sides of eigen-equation (6) and integration by part, one has

$$
\begin{aligned}
\int_{t \leq r \leq r_{j}} r^{1-2 m} q_{0} v_{m}^{2} e^{-2 \rho} d x= & \int_{t \leq r \leq r_{j}}(1-2 m) r^{-2 m} \frac{\partial v_{m}}{\partial r} v_{m} e^{-2 \rho} d x \\
& +\int_{t \leq r \leq r_{j}} r^{1-2 m}\left|\nabla v_{m}\right|^{2} e^{-2 \rho} d x+\int_{t \leq r \leq r_{j}} 2 m r^{-2 m} \frac{\partial v_{m}}{\partial r} v_{m} e^{-2 \rho} d x \\
& +\int_{S_{t}} r^{1-2 m} \frac{\partial v_{m}}{\partial r} v_{m} e^{-2 \rho} d x-\int_{S_{r_{j}}} r^{1-2 m} \frac{\partial v_{m}}{\partial r} v_{m} e^{-2 \rho} d x
\end{aligned}
$$

By (9), one has

$$
\begin{aligned}
\int_{r>t} r^{1-2 m} q_{0} v_{m}^{2} e^{-2 \rho} d x= & \int_{r>t} r^{-2 m} \frac{\partial v_{m}}{\partial r} v_{m} e^{-2 \rho} d x \\
& +\int_{r>t} r^{1-2 m}\left|\nabla v_{m}\right|^{2} e^{-2 \rho} d x+\int_{S_{t}} r^{1-2 m} \frac{\partial v_{m}}{\partial r} v_{m} e^{-2 \rho} d x
\end{aligned}
$$

Now the lemma follows by (10) and (11).

\section{Construction of the energy functions}

In this section, we will give the general construction of energy functions and derive the formulas for their derivatives. Those derivation can be found in [23]. In order to make the readers easy to understand, we give the details here. Let

$$
\begin{aligned}
F(m, r, s)= & r^{s} \int_{S_{r}} \frac{1}{2}\left[\frac{m(m+1)}{r^{2}}+q\right] v_{m}^{2} e^{-2 \rho} d x \\
& \left.+r^{s} \int_{S_{r}}\left[\left|\frac{\partial v_{m}}{\partial r}\right|^{2}-\frac{1}{2}\left|\nabla v_{m}\right|^{2}\right)\right] e^{-2 \rho} d x \\
= & \mathrm{I}+\mathrm{II},
\end{aligned}
$$

where

$$
\begin{aligned}
\mathrm{I} & =r^{s} \int_{S_{r}}\left[\left|\frac{\partial v_{m}}{\partial r}\right|^{2}-\frac{1}{2}\left|\nabla v_{m}\right|^{2}\right] e^{-2 \rho} d x \\
& =\frac{1}{2} r^{s} \int_{S_{r}}\left[\left|\frac{\partial v_{m}}{\partial r}\right|^{2}-\left|\nabla{ }_{\omega} v_{m}\right|^{2}\right] e^{-2 \rho} d x
\end{aligned}
$$

and

$$
\mathrm{II}=\frac{1}{2} r^{s} \int_{S_{r}}\left[\frac{m(m+1)}{r^{2}}+q\right] v_{m}^{2} e^{-2 \rho} d x .
$$

We begin with the derivation of $\frac{\partial}{\partial r} \mathrm{I}$. By Lemma 2.2, one has

$$
\begin{aligned}
\frac{\partial}{\partial r} \mathrm{I}= & s r^{s-1} \int_{S_{r}}\left[\frac{1}{2}\left|\frac{\partial v_{m}}{\partial r}\right|^{2}-\frac{1}{2}\left|\nabla_{\omega} v_{m}\right|^{2}\right] e^{-2 \rho} d x+r^{s} \int_{S_{r}}\left[\frac{\partial v_{m}}{\partial r} \frac{\partial^{2} v_{m}}{\partial r^{2}}-\frac{1}{2} \frac{\partial}{\partial r}\left\langle\nabla_{\omega} v_{m}, \nabla_{\omega} v_{m}\right\rangle\right] e^{-2 \rho} d x \\
& +r^{s} \int_{S_{r}} \frac{1}{2}\left(\Delta r-2 \rho^{\prime}\right)\left[\left|\frac{\partial v_{m}}{\partial r}\right|^{2}-\left|\nabla_{\omega} v_{m}\right|^{2}\right] e^{-2 \rho} d x
\end{aligned}
$$


Using $\Delta v_{m}=\frac{\partial^{2} v_{m}}{\partial r^{2}}+\Delta r \frac{\partial v_{m}}{\partial r}+\Delta_{\omega} v_{m}$, we get

$$
\begin{aligned}
\frac{\partial}{\partial r} \mathrm{I}= & \int_{S_{r}}\left[\frac{s}{2} r^{s-1}\left|\frac{\partial v_{m}}{\partial r}\right|^{2}-2 r^{s} \rho^{\prime}\left|\frac{\partial v_{m}}{\partial r}\right|^{2}+r^{s} \frac{\partial v_{m}}{\partial r} \Delta v_{m}+\frac{r^{s}}{2}\left(2 \rho^{\prime}-\Delta r\right)\left|\frac{\partial v_{m}}{\partial r}\right|^{2}\right] e^{-2 \rho} d x \\
& +r^{s} \int_{S_{r}}\left[\left(-\frac{s}{2 r}+\frac{1}{2}\left(2 \rho^{\prime}-\Delta r\right)\right) \hat{g}\left(\nabla v_{m}, \nabla v_{m}\right)\right] e^{-2 \rho} d x \\
& +r^{s} \int_{S_{r}}\left[\left\langle\nabla_{\omega} \frac{\partial v_{m}}{\partial r}, \nabla_{\omega} v_{m}\right\rangle-\frac{1}{2} \frac{\partial}{\partial r}\left\langle\nabla_{\omega} v_{m}, \nabla_{\omega} v_{m}\right\rangle\right] e^{-2 \rho} d x
\end{aligned}
$$

By some basic computations, one has

$$
\begin{aligned}
\left\langle\nabla_{\omega} \frac{\partial v_{m}}{\partial r}, \nabla_{\omega} v_{m}\right\rangle-\frac{1}{2} \frac{\partial}{\partial r}\left\langle\nabla_{\omega} v_{m}, \nabla_{\omega} v_{m}\right\rangle & =\left\langle\nabla_{\omega} \frac{\partial v_{m}}{\partial r}, \nabla_{\omega} v_{m}\right\rangle-\left\langle\nabla_{\frac{\partial}{\partial r}} \nabla_{\omega} v_{m}, \nabla_{\omega} v_{m}\right\rangle \\
& =(\nabla d r)\left(\nabla_{\omega} v_{m}, \nabla_{\omega} v_{m}\right) .
\end{aligned}
$$

Finally, we get

$$
\begin{aligned}
\frac{\partial}{\partial r} \mathrm{I}= & \int_{S_{r}}\left[\frac{s}{2} r^{s-1}\left|\frac{\partial v_{m}}{\partial r}\right|^{2}-2 r^{s} \rho^{\prime}\left|\frac{\partial v_{m}}{\partial r}\right|^{2}+r^{s} \frac{\partial v_{m}}{\partial r} \Delta v_{m}+\frac{r^{s}}{2}\left(2 \rho^{\prime}-\Delta r\right)\left|\frac{\partial v_{m}}{\partial r}\right|^{2}\right] e^{-2 \rho} d x \\
& +r^{s} \int_{S_{r}}\left[\left(\nabla d r+\left(-\frac{s}{2 r}+\frac{1}{2}\left(2 \rho^{\prime}-\Delta r\right)\right) \hat{g}\right)\left(\nabla v_{m}, \nabla v_{m}\right)\right] e^{-2 \rho} d x .
\end{aligned}
$$

Now we are in the position to obtain $\frac{\partial}{\partial r}$ II. By Lemma 2.2 again, one has

$$
\begin{aligned}
\frac{\partial}{\partial r} \mathrm{II}= & \int_{S_{r}}\left[\frac{\partial}{\partial r} \frac{r^{s}}{2}\left(\frac{m(m+1)}{r^{2}}+q\right) v_{m}^{2}\right] e^{-2 \rho} d x \\
& +\int_{S_{r}}\left(\Delta r-2 \rho^{\prime}\right) \frac{r^{s}}{2}\left[\frac{m(m+1)}{r^{2}}+q\right] v_{m}^{2} e^{-2 \rho} d x \\
= & \int_{S_{r}}\left[\frac{s-2}{2} r^{s-3} m(m+1)+\frac{r^{s}}{2} \frac{\partial q}{\partial r}+\frac{s}{2} r^{s-1} q\right] v_{m}^{2} e^{-2 \rho} d x \\
& +r^{s} \int_{S_{r}}\left[\frac{m(m+1)}{r^{2}}+q\right] v_{m} \frac{\partial v_{m}}{\partial r} e^{-2 \rho} d x \\
& +\frac{r^{s}}{2} \int_{S_{r}}\left(\Delta r-2 \rho^{\prime}\right)\left[\frac{m(m+1)}{r^{2}}+q\right] v_{m}^{2} e^{-2 \rho} d x
\end{aligned}
$$

Putting (13) and (14) together and using (6), we obtain

$$
\begin{aligned}
\frac{\partial F(m, r, s)}{\partial r}= & r^{s-1} \int_{S_{r}}\left[\left(r(\nabla d r)-\left(\frac{s}{2}+\frac{\bar{\delta}}{2}\right) \hat{g}\right)\left(\nabla v_{m}, \nabla v_{m}\right)\right] e^{-2 \rho} d x \\
& +r^{s-1} \int_{S_{r}}\left[2 m-\frac{\bar{\delta}}{2}+\frac{s}{2}\right]\left|\frac{\partial v_{m}}{\partial r}\right|^{2} e^{-2 \rho} d x \\
& +r^{s-1} \int_{S_{r}}\left[r\left(V_{0}+V_{1}+V_{2}+q-\lambda\right)+\frac{m}{r} \bar{\delta}\right] \frac{\partial v_{m}}{\partial r} v_{m} e^{-2 \rho} d x \\
& +r^{s-1} \int_{S_{r}}\left[\frac{s-2}{2} \frac{m(m+1)}{r^{2}}+\frac{1}{2} r \frac{\partial q}{\partial r}+\frac{s}{2} q\right] v_{m}^{2} e^{-2 \rho} d x \\
& +r^{s-1} \int_{S_{r}} \frac{1}{2} \bar{\delta}\left[\frac{m(m+1)}{r^{2}}+q\right] v_{m}^{2} e^{-2 \rho} d x .
\end{aligned}
$$

Let $m=0$ and $F(r, s)=F(0, r, s)$, we obtain 


$$
\begin{aligned}
\frac{\partial F(r, s)}{\partial r}= & r^{s-1} \int_{S_{r}}\left[\left(r(\nabla d r)-\left(\frac{s}{2}+\frac{1}{2} \bar{\delta}\right) \hat{g}\right)(\nabla v, \nabla v)\right] e^{-2 \rho} d x \\
& +r^{s-1} \int_{S_{r}}\left[-\frac{\bar{\delta}}{2}+\frac{s}{2}\right]\left|\frac{\partial v}{\partial r}\right|^{2} e^{-2 \rho} d x \\
& +r^{s-1} \int_{S_{r}}\left[r\left(V_{0}+V_{1}+V_{2}+q-\lambda\right)\right] \frac{\partial v}{\partial r} v e^{-2 \rho} d x \\
& +r^{s-1} \int_{S_{r}}\left[\frac{1}{2} r \frac{\partial q}{\partial r}+\frac{s}{2} q+\frac{\bar{\delta}}{2} q\right] v^{2} e^{-2 \rho} d x .
\end{aligned}
$$

\section{Exponential DECAY of the Eigensolutions}

In this section, we will prove the exponential decay of the eigensolution based on the weakly exponential decay assumption and then show such eigensolution is trivial.

Theorem 4.1. Suppose

$$
\int_{r \geq r_{0}}\left(|\nabla u|^{2}+u^{2}\right) e^{c \sqrt{r}} d x<\infty
$$

for some $c>0$, then

$$
\int_{r \geq r_{0}}\left(|\nabla u|^{2}+u^{2}\right) e^{c r} d x<\infty
$$

for some $c>0$.

Proof. By the assumption, we have

$$
\int_{r \geq r_{0}}\left(|\nabla v|^{2}+v^{2}\right) e^{c \sqrt{r}} e^{-2 \rho} d x<\infty
$$

for some $c>0$. Let $q_{1}=\lambda-\frac{b^{2}}{4}-\frac{b c}{2 r}-V_{2}$ in the energy function (12). By (15), we have

$$
\begin{aligned}
& \frac{\partial F(m, r, s)}{\partial r}+\alpha \frac{\partial}{\partial r} \int_{S_{r}} r^{s-1} v_{m}^{2} e^{-2 \rho} d x \\
= & r^{s-1} \int_{S_{r}}\left[\left(r(\nabla d r)-\left(\frac{s}{2}+\frac{1}{2} \bar{\delta}\right) \hat{g}\right)\left(\nabla v_{m}, \nabla v_{m}\right)\right] e^{-2 \rho} d x \\
+ & r^{s-1} \int_{S_{r}}\left[2 m-\frac{\bar{\delta}}{2}+\frac{s}{2}\right]\left|\frac{\partial v_{m}}{\partial r}\right|^{2} e^{-2 \rho} d x \\
+ & r^{s-1} \int_{S_{r}}\left[\frac{\bar{\delta} b}{2}+\bar{\delta} \frac{m}{r}+2 \alpha+o(1)\right] \frac{\partial v_{m}}{\partial r} v_{m} e^{-2 \rho} d x \\
+ & r^{s-1} \int_{S_{r}}\left[\left(\lambda-\frac{b^{2}}{4}\right)\left(\frac{s}{2}+\frac{\bar{\delta}}{2}\right)+o(1)\right] v_{m}^{2} e^{-2 \rho} d x \\
+ & r^{s-1} \int_{S_{r}} \frac{m(m+1)}{r^{2}}\left[\frac{s-2}{2}+\frac{\bar{\delta}}{2}\right] v_{m}^{2} e^{-2 \rho} d x .
\end{aligned}
$$

By the assumption (17), for any $m$, there exists a sequence $r_{j}$ such that

$$
F\left(m, r_{j}, s\right)+\alpha \int_{S_{r_{j}}} r^{s-1} v_{m}^{2} e^{-2 \rho} d x=o(1) .
$$


By assumption (21), there exists $0<s_{0}<1$ such that

$$
r(\nabla d r)-\left(\frac{s_{0}}{2}+\frac{1}{2} \bar{\delta}\right)>0 .
$$

By the Cauchy Schwartz inequality, one has

$$
(\underline{201})+(21)+(221)+(23) \geq r^{s-1} \int_{S_{r}}\left[\epsilon-C_{1} \frac{m^{2}}{r^{2}}\right] v_{m}^{2} e^{-2 \rho} d x,
$$

for large $m$ and $r$. Combining with (18) and (24), we have

$$
-F\left(m, r, s_{0}\right)-\alpha \int_{S_{r}} r^{s_{0}-1} v_{m}^{2} e^{-2 \rho} d x \geq \int_{r(x)>r} r(x)^{s_{0}-1}\left[\epsilon-C_{1} \frac{m^{2}}{r(x)^{2}}\right] v_{m}^{2} e^{-2 \rho} d x .
$$

That is

$$
\begin{gathered}
r^{s_{0}} \int_{S_{r}}\left[\frac{1}{2}\left|\nabla v_{m}\right|^{2}-\left|\frac{\partial v_{m}}{\partial r}\right|^{2}\right] e^{-2 \rho} d x-r^{s_{0}} \int_{S_{r}} \frac{1}{2}\left[\frac{m(m+1)}{r^{2}}+q_{1}\right] v_{m}^{2} e^{-2 \rho} d x-\alpha \int_{S_{r}} r^{s_{0}-1} v_{m}^{2} e^{-2 \rho} d x \\
\geq \int_{r(x)>r} r(x)^{s_{0}-1}\left[\epsilon-C_{1} \frac{m^{2}}{r(x)^{2}}\right] v_{m}^{2} e^{-2 \rho} d x .
\end{gathered}
$$

Multiplying both side of (25) by $r^{1-s_{0}-2 m}$ and integrating it with respect to $r$ over $[t, \infty)$, we have

$$
\begin{gathered}
\int_{r>t} r^{1-2 m}\left[\left|\nabla v_{m}\right|^{2}-\left(\frac{m(m+1)}{r^{2}}+q_{1}\right) v_{m}^{2}\right] e^{-2 \rho} d x-2 \alpha \int_{r>t} r^{-2 m} v_{m}^{2} e^{-2 \rho} d x-2 \int_{r>t} r^{1-2 m}\left|\frac{\partial v_{m}}{\partial r}\right|^{2} e^{-2 \rho} d x \\
\geq \int_{r>t} r^{1-s_{0}-2 m} d r \int_{r(x)>r} r(x)^{s_{0}-1}\left[\epsilon-C_{1} \frac{m^{2}}{r(x)^{2}}\right] v_{m}^{2} e^{-2 \rho} d x \\
\quad \geq\left[\epsilon-C_{1} \frac{m^{2}}{t^{2}}\right] \int_{r>t} r^{1-s_{0}-2 m} d r \int_{r(x)>r} r(x)^{s_{0}-1} v_{m}^{2} e^{-2 \rho} d x
\end{gathered}
$$

By Cauchy Schwartz inequality again, one has

$$
\left|\int_{r>t} r^{-2 m} \frac{\partial v_{m}}{\partial r} v_{m} e^{-2 \rho} d x\right| \leq \int_{r>t} \frac{1}{2} r^{-2 m}\left[\left|\frac{\partial v_{m}}{\partial r}\right|^{2}+v_{m}^{2}\right] e^{-2 \rho} d x .
$$

By Lemma 2.3. one has

$$
\begin{aligned}
\int_{r>t} r^{1-2 m}\left[\left|\nabla v_{m}\right|^{2}-q_{0} v_{m}^{2}\right] e^{-2 \rho} d x= & -\frac{1}{2} \frac{d}{d t}\left(t^{1-2 m} \int_{S_{t}} v_{m}^{2} e^{-2 \rho} d x\right)-\frac{1}{2} \int_{S_{t}} r^{-2 m}(2 m-1) v_{m}^{2} e^{-2 \rho} d x \\
& +\frac{1}{2} \int_{S_{t}} t^{1-2 m}\left(\Delta r-2 \rho^{\prime}\right) v_{m}^{2} e^{-2 \rho} d x-\int_{r>t} r^{-2 m} \frac{\partial v_{m}}{\partial r} v_{m} e^{-2 \rho} d x,
\end{aligned}
$$

where $q_{0}=\lambda-V_{0}-V_{1}-V_{2}+\frac{m(m+1)}{r^{2}}+\frac{m}{r}\left(2 \rho^{\prime}-\Delta r\right)$.

By the definition of $q_{0}$ and $q_{1}$, we have

$$
\left|q_{0}-q_{1}-\frac{m(m+1)}{r^{2}}\right| \leq 2 \frac{m}{r^{2}}|\delta|+O\left(\frac{1}{r}\right) .
$$

By (26), (27), (28) and (29), we have

$$
\begin{gathered}
-\frac{1}{2} \frac{d}{d t}\left(t^{1-2 m} \int_{S_{t}} v_{m}^{2} e^{-2 \rho} d x\right)-\frac{1}{4} \int_{S_{t}} t^{-2 m}(2 m-1) v_{m}^{2} e^{-2 \rho} d x-\frac{1}{2} \alpha \int_{r>t} r^{-2 m} v_{m}^{2} e^{-2 \rho} d x+C_{2} \int_{r>t} r^{-2 m} \frac{m}{r} v_{m}^{2} e^{-2 \rho} d x \\
(30) \quad \geq\left[\epsilon-C_{1} \frac{m^{2}}{t^{2}}\right] \int_{r>t} r^{1-s_{0}-2 m} d r \int_{r(x)>r} r(x)^{s_{0}-1} v_{m}^{2} e^{-2 \rho} d x,
\end{gathered}
$$


for large $m$ and $t$. Notice that $C_{1}$ and $C_{2}$ do not depend on $\alpha$. For any large $t$, let $m$ be such that

$$
\epsilon-C_{1} \frac{m^{2}}{t^{2}}=0 .
$$

That is $\frac{m}{t}=\sqrt{\frac{\epsilon}{C_{1}}}$.

Let $\alpha$ be large enough so that

$$
-\frac{1}{2} \alpha \int_{r>t} r^{-2 m} v_{m}^{2} e^{-2 \rho} d x+C_{2} \int_{r>t} r^{-2 m} \frac{m}{r} v_{m}^{2} e^{-2 \rho} d x<0,
$$

since $\frac{m}{r} \leq \frac{m}{t}$ for $r \geq t$. Thus, (30) leads to

$$
-\frac{1}{2} \frac{d}{d t}\left(t^{1-2 m} \int_{S_{t}} v_{m}^{2} e^{-2 \rho} d x\right)-\frac{1}{4} \frac{m}{t} \int_{S_{t}} t^{1-2 m} v_{m}^{2} e^{-2 \rho} d x<0 .
$$

By the fact that $\frac{m}{t}=\sqrt{\frac{\epsilon}{C_{1}}}$, we have

$$
-\frac{d}{d t}\left(t^{1-2 m} \int_{S_{t}} v_{m}^{2} e^{-2 \rho} d x\right)-\epsilon \int_{S_{t}} t^{1-2 m} v_{m}^{2} e^{-2 \rho} d x \geq 0
$$

for some $\epsilon>0$. Let

$$
G(t)=t^{1-2 m} \int_{S_{t}} v_{m}^{2} e^{-2 \rho} d x=t \int_{S_{t}} v^{2} e^{-2 \rho} d x
$$

So (31) becomes

This implies

$$
\frac{d G}{d t} \leq-\epsilon G(t)
$$

$$
G(t) \leq e^{-\epsilon t}
$$

for some $\epsilon>0$. Thus, we get

$$
\int_{S_{r}} v^{2} e^{-2 \rho} d x \leq e^{-\epsilon r}
$$

We will show that

$$
\int_{r \geq r_{0}}|\nabla v| e^{-2 \rho} e^{\epsilon r} d x<\infty
$$

for some $\epsilon>0$. By Cauchy Schwartz inequality and (17), one has

$$
\int_{r \geq r_{0}}\left|\frac{\partial v}{\partial r} v\right| e^{\epsilon r} e^{-2 \rho} d x<\infty
$$

Thus there exists sequence $r_{j}$ such that

$$
\int_{S_{r_{j}}}\left|\frac{\partial v}{\partial r} v\right| e^{\epsilon r} e^{-2 \rho} d x=o(1) .
$$

Using the eigenequation (5) and integration by part, one has

$$
\int_{r_{0} \leq r \leq r_{j}}|\nabla v|^{2} e^{\epsilon r} e^{-2 \rho} d x+\int_{S_{r}} \frac{\partial v}{\partial r} v e^{\epsilon r} e^{-2 \rho} d x-\int_{S_{r_{j}}} \frac{\partial v}{\partial r} v e^{\epsilon r} e^{-2 \rho} d x<\infty,
$$

since (32) and (34) hold.

By letting $j \rightarrow \infty$ in (35), we obtain

$$
\int_{r>r_{0}}|\nabla v|^{2} e^{\epsilon r} e^{-2 \rho} d x<\infty
$$


We finish the proof.

Theorem 4.2. Suppose

$$
\int_{r \geq r_{0}}\left(|\nabla v|^{2}+v^{2}\right) e^{c r^{\sigma}} e^{-2 \rho} d x<\infty
$$

for any $\sigma<1$, then $v \equiv 0$.

Proof. Let $\bar{v}=e^{k r^{\theta}} v$ and $\theta$ be close to 1 . In order to write down our proof smoothly, let $\bar{\rho}=k r^{\theta}$. Direct computation implies

$$
\Delta v=e^{-\bar{\rho}} \Delta \bar{v}-2 \bar{\rho}^{\prime} e^{-\bar{\rho}} \frac{\partial \bar{v}}{\partial r}+\left(\bar{\rho}^{\prime 2}-\bar{\rho}^{\prime \prime}-\bar{\rho}^{\prime} \Delta r\right) e^{-\bar{\rho}} \bar{v} .
$$

By (5), we get the equation of $\bar{v}$,

$$
\Delta \bar{v}=2\left(\bar{\rho}^{\prime}+\rho^{\prime}\right) \frac{\partial \bar{v}}{\partial r}+\left(V_{1}+V_{2}+V_{0}+\bar{V}_{0}-\lambda\right) \bar{v},
$$

where

$$
\begin{aligned}
\bar{V}_{0} & =-\bar{\rho}^{2}+\bar{\rho}^{\prime} \Delta r+\bar{\rho}^{\prime \prime}-2 \rho^{\prime} \bar{\rho}^{\prime} \\
& =-k^{2} \theta^{2} r^{2 \theta-2}-k(1-\theta) \theta r^{\theta-2}+k r^{\theta-1}\left(\Delta r-2 \rho^{\prime}\right) .
\end{aligned}
$$

Define the new energy function of $\bar{v}$ as follows

$$
\bar{F}(r)=\int_{S_{r}}\left[\frac{1}{2} \bar{q} \bar{v}^{2}+\left|\frac{\partial \bar{v}}{\partial r}\right|^{2}-\frac{1}{2}|\nabla \bar{v}|^{2}\right] e^{-2 \rho} d x,
$$

where $\bar{q}$ will be determined later. By the assumption (2), there exists some $s_{0}$ (actually we can let $s_{0}$ be close to $\delta$ and $s_{0}>\delta$ ) such that

$$
\left|r\left(\Delta r-2 \rho^{\prime}\right)+s_{0}\right|<2 a
$$

and

$$
r\left(\Delta r-2 \rho^{\prime}\right)+s_{0}>\gamma>0 .
$$

By the similar argument of (15) or (16), one has

$$
\begin{aligned}
\frac{\partial r^{s_{0}} \bar{F}(r)}{\partial r}= & \int_{S_{r}} r^{s_{0}}\left[\left(\nabla d r-\left(\frac{s}{2 r}+\frac{1}{2}\left(\Delta r-2 \rho^{\prime}\right)\right) \hat{g}\right)(\nabla \bar{v}, \nabla \bar{v})\right] e^{-2 \rho} d x \\
& +\int_{S_{r}} r^{s_{0}}\left[\bar{q} \bar{v} \frac{\partial \bar{v}}{\partial r}+\frac{1}{2} \frac{\partial \bar{q}}{\partial r} \bar{v}^{2}+\frac{\partial \bar{v}}{\partial r}\left(\Delta \bar{v}-\Delta r \frac{\partial \bar{v}}{\partial r}\right)+\left(\Delta r-2 \rho^{\prime}\right)\left(\frac{1}{2} \bar{q} \bar{v}^{2}+\frac{1}{2}\left|\frac{\partial \bar{v}}{\partial r}\right|^{2}\right)\right] e^{-2 \rho} d x \\
& +s_{0} r^{s_{0}-1} \int_{S_{r}}\left[\frac{1}{2} \bar{q} \bar{v}^{2}+\frac{1}{2}\left|\frac{\partial \bar{v}}{\partial r}\right|^{2}\right] e^{-2 \rho} d x \\
(38)= & r^{s_{0}-1} \int_{S_{r}}\left[\left(r \nabla d r-\left(\frac{s_{0}}{2}+\frac{1}{2} \bar{\delta}\right) \hat{g}\right)(\nabla \bar{v}, \nabla \bar{v})\right] e^{-2 \rho} d x \\
& +r^{s_{0}-1} \int_{S_{r}}\left[-\frac{\delta}{2}+\frac{s_{0}}{2}+2 r \bar{\rho}^{\prime}\right]\left|\frac{\partial \bar{v}}{\partial r}\right|^{2} e^{-2 \rho} d x \\
& +r^{s_{0}-1} \int_{S_{r}} r\left[\bar{q}+V_{1}+V_{2}+V_{0}+\bar{V}_{0}-\lambda\right] \frac{\partial \bar{v}}{\partial r} \bar{v} e^{-2 \rho} d x \\
& +r^{s_{0}-1} \int_{S_{r}}\left[\frac{r}{2} \frac{\partial \bar{q}}{\partial r}+\frac{1}{2} \bar{\delta} \bar{q}+\frac{s_{0}}{2} \bar{q}\right] \bar{v}^{2} e^{-2 \rho} d x .
\end{aligned}
$$


Let $\bar{q}=\lambda-\frac{b^{2}}{4}-V_{2}+k^{2} \theta^{2} r^{2 \theta-2}+k \theta(1-\theta) r^{\theta-2}$. By (38), one has

$$
\begin{aligned}
\frac{\partial r^{s_{0}} \bar{F}}{\partial r}= & r^{s_{0}-1} \int_{S_{r}}\left[\left(r \nabla d r-\left(\frac{s_{0}}{2}+\frac{1}{2} \bar{\delta}\right) \hat{g}\right)(\nabla \bar{v}, \nabla \bar{v})\right] e^{-2 \rho} d x \\
& +r^{s_{0}-1} \int_{S_{r}}\left[2 k \theta r^{\theta}+O(1)\right]\left|\frac{\partial \bar{v}}{\partial r}\right|^{2} e^{-2 \rho} d x \\
& +r^{s_{0}-1} \int_{S_{r}}\left[O(1)+k \bar{\delta} r^{\theta-1}\right] \frac{\partial \bar{v}}{\partial r} \bar{v} e^{-2 \rho} d x \\
& +r^{s_{0}-1} \int_{S_{r}}\left[o(1)+k^{2} \theta^{2}(\theta-1) r^{2 \theta-2}+\frac{1}{2} k \theta(1-\theta)(\theta-2) r^{\theta-2}\right. \\
& \left.+\frac{1}{2}\left(\bar{\delta}+s_{0}\right)\left(\epsilon+o(1)+k(1-\theta) \theta r^{\theta-2}+k^{2} \theta^{2} r^{2 \theta-2}\right)\right] \bar{v}^{2} e^{-2 \rho} d x
\end{aligned}
$$

with some $\epsilon=\lambda-\frac{b^{2}}{4}>0$. By the choice that $\theta$ is close to 1 , one has

$$
\text { (411) }+ \text { (42) }>0
$$

for large $r$ and $k$.

By Cauchy Schwartz inequality, we have

$$
|(40)|<(39)+(41)+(42) \text {. }
$$

Thus

$$
\frac{\partial r^{s_{0}} \bar{F}(r)}{\partial r}>0
$$

for large $k$ (only depends on the bounds of constants in the assumptions of Theorem 1.1).

By the assumption of (36), there exists sequence $r_{j}$ going to infinity such that

$$
\lim _{j} r_{j}^{s_{0}} \bar{F}\left(r_{j}\right)=0 .
$$

By the fact that $r^{s_{0}} \bar{F}(r)$ is monotone (using (43)), we have

$$
\bar{F}(r) \leq 0
$$

for $r>R$. Note that $R$ does not depend on $k$. Fix $r>R$, by (37), one has

$$
\bar{F}(r)=e^{2 k r^{\theta}} \int_{S_{r}}\left[\frac{1}{2}\left(\bar{q}+k^{2} \theta^{2} r^{2 \theta-2}\right) v^{2}+\left|\frac{\partial v}{\partial r}\right|^{2}+k \theta r^{\theta-1} \frac{\partial v}{\partial r} v-\frac{1}{2}|\nabla v|\right] e^{-2 \rho} d x \leq 0,
$$

for all large $k$ and large $r$. This is impossible except

$$
\int_{S_{r}}|v|^{2} e^{-2 \rho} d x=0
$$

for all large $r$. By unique continuation theorem, we have $v \equiv 0$.

\section{The positivity of initial energy and proof of Theorem 1.1}

Theorem 5.1. Suppose

$$
q-\lambda+V_{0}+V_{1}+V_{2} \geq \frac{\epsilon}{r},
$$

for some $\epsilon>0$. We also assume

$$
\liminf _{r} \int_{S_{r}}\left|\frac{\partial v}{\partial r} v\right| e^{-2 \rho} d x=0
$$


Then for any $R>0$, there exists $r>R$ such that

$$
F(r, 0)>0 \text {. }
$$

Proof. Otherwise $F(r, 0) \leq 0$ for all $r>R$.

By the assumption (45), there exists a sequence $r_{j}$ going to infinity such that

$$
\int_{S_{r_{j}}}\left|\frac{\partial v}{\partial r} v\right| e^{-2 \rho} d x=o(1)
$$

Integrating $F(r, 0)$ from $r$ to $r_{j}$, one has

$$
\int_{r \leq r(x) \leq r_{j}}\left[q v^{2}+\left|\frac{\partial v}{\partial r}\right|^{2}-\left|\nabla_{\omega} v\right|^{2}\right] e^{-2 \rho} d x \leq 0 .
$$

Using the eigen-equation (5) and integration by part, one has

$$
\int_{r \leq r(x) \leq r_{j}}|\nabla v|^{2} e^{-2 \rho} d x+\int_{S_{r}} \frac{\partial v}{\partial r} v e^{-2 \rho} d x-\int_{S_{r_{j}}} \frac{\partial v}{\partial r} v e^{-2 \rho} d x=\int_{r \leq r(x) \leq r_{j}}\left[\lambda-V_{0}-V_{1}-V_{2}\right] v^{2} e^{-2 \rho} d x .
$$

Putting (46) and (48) into (47), and letting $r_{j}$ go to infinity, we have

$$
\int_{S_{r}} \frac{\partial v}{\partial r} v e^{-2 \rho} d x+\int_{r(x) \geq r}\left[\left(q-\lambda+V_{0}+V_{1}+V_{2}\right) v^{2}+2\left|\frac{\partial v}{\partial r}\right|^{2}\right] e^{-2 \rho} d x \leq 0 .
$$

By the assumption (44), we have

$$
\int_{S_{r}} \frac{\partial v}{\partial r} v e^{-2 \rho} d x+\int_{r(x) \geq r}\left[\frac{\epsilon}{r} v^{2}+2\left|\frac{\partial v}{\partial r}\right|^{2}\right] e^{-2 \rho} d x \leq 0 .
$$

Let

$$
G(r)=\int_{r(x) \geq r}\left[\frac{\epsilon}{r} v^{2}+2\left|\frac{\partial v}{\partial r}\right|^{2}\right] e^{-2 \rho} d x .
$$

By (49) and Cauchy-Schwartz inequality, one has

$$
G(r) \leq-C \sqrt{r} \frac{d G}{d r}
$$

where $C>0$ is a constant depending on $\epsilon$. Solving (50), one has

$$
G(r) \leq e^{-c \sqrt{r}}
$$

for large $r$. This yields that

$$
\int_{r \geq r_{0}}\left[v^{2}+2\left|\frac{\partial v}{\partial r}\right|^{2}\right] e^{c \sqrt{r}} e^{-2 \rho} d x<\infty
$$

for some $c>0$, and

$$
\int_{r \geq r_{0}}\left|v \frac{\partial v}{\partial r}\right| e^{c \sqrt{r}} e^{-2 \rho} d x<\infty .
$$

Multiplying $e^{c \sqrt{r}} v e^{-2 \rho}$ in (15) and integration by part, one has

$$
\int_{r \geq r_{0}}|\nabla v|^{2} e^{c \sqrt{r}} e^{-2 \rho} d x<\infty
$$

By Theorems 4.1, 4.2 and (51), (52), we have $v \equiv 0$, which is contradicted to our assumption. 
Recall that

$$
\bar{\delta}(r)=r\left(\Delta r-b-\frac{c}{r}\right) .
$$

Let $q=\lambda-\frac{b^{2}}{4}-\frac{b c}{2 r}+\frac{b(\delta \pm \epsilon)}{2 r}-V_{2}$, where \pm is defined such that $b( \pm \epsilon)>0$. Thus (44) holds. Now by (16), the derivative of the energy function for $v$ has the following form,

$$
\begin{aligned}
\frac{\partial F(r, s)}{\partial r}= & \left.r^{s-1} \int_{S_{r}}\left[r(\nabla d r)-\left(\frac{s}{2}+\frac{1}{2} \bar{\delta}\right) \hat{g}\right)(\nabla v, \nabla v)\right] e^{-2 \rho} d x \\
& +r^{s-1} \int_{S_{r}}\left[-\frac{\bar{\delta}}{2}+\frac{s}{2}+o(1)\right]\left|\frac{\partial v}{\partial r}\right|^{2} e^{-2 \rho} d x \\
& +r^{s-1} \int_{S_{r}}\left[\frac{\bar{\delta} b}{2}+\frac{(\delta \pm \epsilon) b}{2}\right] \frac{\partial v}{\partial r} v e^{-2 \rho} d x \\
& +r^{s-1} \int_{S_{r}}\left[\left(\lambda-\frac{b^{2}}{4}\right)\left(\frac{s}{2}+\frac{\bar{\delta}}{2}\right)+o(1)\right] v^{2} e^{-2 \rho} d x
\end{aligned}
$$

Theorem 5.2. Let $s<\mu$ and $s$ be sufficiently close to $\mu$. Then under the assumptions of Theorem 1.1, we have

$$
\frac{\partial F(r, s)}{\partial r}>0
$$

for large $r$.

Proof. By the assumptions of Theorem 1.1 and $s$ is close to $\mu$, one has

$$
|\bar{\delta}+s|<2 a
$$

and

$$
\bar{\delta}+s>0 .
$$

By Cauchy Schwartz inequality and (53)-(56), it suffices to prove

$$
4\left[\left(\lambda-\frac{b^{2}}{4}\right)\left(\frac{s}{2}+\frac{\bar{\delta}}{2}\right)\right]\left[-\frac{\bar{\delta}}{2}+\frac{s}{2}\right]>\left|\frac{\bar{\delta} b}{2}+\frac{(\delta \pm \epsilon) b}{2}\right|^{2} .
$$

Solving inequality (57), we get

$$
\lambda>\frac{b^{2}}{4}+\frac{1}{4} \frac{(\bar{\delta}+\delta \pm \epsilon)^{2} b^{2}}{s^{2}-\bar{\delta}^{2}} .
$$

It is clear that (58) holds if

$$
\lambda>\frac{b^{2}}{4}+\frac{\delta^{2} b^{2}}{s^{2}-\delta^{2}},
$$

since $\epsilon$ is small. This follows from the assumption (3) and the fact that $s$ is close to $\mu$.

\section{Proof of Theorem 1.1}

Proof. It suffices to assume

$$
\liminf _{r} \int_{S_{r}}\left|\frac{\partial v}{\partial r} v\right| e^{-2 \rho} d x=0
$$

so that (45) holds. By Theorem 5.1, there exists a sequence $r_{n}$ such that

$$
F\left(r_{n}, 0\right)>0 .
$$

By Theorem [5.2, there exists $\gamma>0$ such that

$$
F(r, s) \geq \gamma,
$$


for large $r$. By the fact $v=e^{\rho} u$, we get that

$$
\liminf _{r \rightarrow \infty} r^{s}\left[M(r)^{2}+N(r)^{2}\right]>0
$$

Recall that $s<\mu$, we have

$$
\liminf _{r \rightarrow \infty} r^{\mu}\left[M(r)^{2}+N(r)^{2}\right]=\infty
$$

\section{ACKNOWLEDGMENTS}

I would like to thank Svetlana Jitomirskaya for introducing to me paper [18] and inspiring discussions on this subject. I would also like to thank the anonymous referee for careful reading of the manuscript that has led to an important improvement, in particular, telling me the work of Ito and Skibsted. Finally, I thank Professor Ito for helpful discussions. The author was supported by the AMS-Simons Travel Grant 2016-2018 and NSF DMS-1700314. This research was also partially supported by NSF DMS-1401204.

\section{REFERENCES}

[1] S. A. Denisov and A. Kiselev. Spectral properties of Schrödinger operators with decaying potentials. In Spectral theory and mathematical physics: a Festschrift in honor of Barry Simon's 60th birthday, volume 76 of Proc. Sympos. Pure Math., pages 565-589. Amer. Math. Soc., Providence, RI, 2007.

[2] H. Donnelly. Negative curvature and embedded eigenvalues. Math. Z., 203(2):301-308, 1990.

[3] H. Donnelly. Spectrum of the Laplacian on asymptotically Euclidean spaces. Michigan Math. J., 46(1):101-111, 1999.

[4] H. Donnelly. Spectral theory of complete Riemannian manifolds. Pure Appl. Math. Q., 6(2, Special Issue: In honor of Michael Atiyah and Isadore Singer):439-456, 2010.

[5] H. Donnelly and N. Garofalo. Riemannian manifolds whose Laplacians have purely continuous spectrum. Math. Ann., 293(1):143-161, 1992.

[6] H. Donnelly and P. Li. Pure point spectrum and negative curvature for noncompact manifolds. Duke Math. J, 46(3):497-503, 1979 .

[7] J. F. Escobar and A. Freire. The spectrum of the Laplacian of manifolds of positive curvature. Duke Math. J, 65(1):1-21, 1992.

[8] R. E. Greene and H. Wu. Function theory on manifolds which possess a pole, volume 699 of Lecture Notes in Mathematics. Springer, Berlin, 1979.

[9] K. Ito and E. Skibsted. Time-dependent scattering theory on manifolds, III. Preprint.

[10] K. Ito and E. Skibsted. Absence of embedded eigenvalues for Riemannian Laplacians. Adv. Math., 248:945-962, 2013.

[11] K. Ito and E. Skibsted. Scattering theory for Riemannian Laplacians. J. Funct. Anal., 264(8):1929-1974, 2013.

[12] K. Ito and E. Skibsted. Stationary scattering theory on manifolds, I. arXiv preprint arXiv:1602.07488. 2016.

[13] K. Ito and E. Skibsted. Stationary scattering theory on manifolds, II. arXiv preprint arXiv:1602.07487. 2016.

[14] S. Jitomirskaya and W. Liu. Noncompact complete Riemannian manifolds with dense eigenvalues embedded in the essential spectrum of the Laplacian. arXiv preprint arXiv:1805.01072

[15] S. Jitomirskaya and W. Liu. Noncompact complete Riemannian manifolds with singular continuous spectrum embedded in the essential spectrum of the Laplacian. Preprint.

[16] T. Kato. Growth properties of solutions of the reduced wave equation with a variable coefficient. Comm. Pure Appl. Math., 12:403-425, 1959.

[17] H. Kumura. On the essential spectrum of the Laplacian on complete manifolds. J. Math. Soc. Japan, 49(1):1-14, 1997.

[18] H. Kumura. The radial curvature of an end that makes eigenvalues vanish in the essential spectrum. I. Math. Ann., 346(4):795-828, 2010.

[19] H. Kumura. The lower bound of the Ricci curvature that yields an infinite discrete spectrum of the Laplacian. Ann. Inst. Fourier (Grenoble), 61(4):1557-1572 (2012), 2011. 
[20] H. Kumura. The radial curvature of an end that makes eigenvalues vanish in the essential spectrum II. Bull. Lond. Math. Soc., 43(5):985-1003, 2011.

[21] H. Kumura. Perturbation of a warped product metric of an end and the growth property of solutions to eigenvalue equations. Kyoto J. Math., 52(2):249-276, 2012.

[22] H. Kumura. Limiting absorption principle on manifolds having ends with various measure growth rate limits. Proc. Lond. Math. Soc. (3), 107(3):517-548, 2013.

[23] W. Liu. The growth of the eigen-solutions of Laplacian on Riemannian manifolds I: construction of energy function. IMRN to appear.

[24] M. A. Pinsky. Spectrum of the Laplacian on a manifold of negative curvature. II. J. Differential Geom., 14(4):609-620 (1981), 1979.

(Wencai Liu) Department of Mathematics, University of California, Irvine, California 92697-3875, USA

E-mail address: liuwencai1226@gmail.com 